\title{
CHILOÉ, UNA SOCIEDAD RURAL IGUALITARIA. DISTRIBUCIÓN DEL INGRESO AGRÍCOLA Y DE LA TIERRA DESPUÉS DE LA INDEPENDENCIA
}

\author{
MANUEL LLORCA-JAÑA ${ }^{a}$, CLAUDIO ROBLES ORTIZ ${ }^{\text {b \& JUAN NAVARRETE-MONTALVOc }}$
}

\begin{abstract}
RESUMEN
Este artículo analiza la distribución del ingreso agrícola y de la tierra en Chiloé entre c.1830 y los años 1850, comparando ambas variables con el resto de las provincias chilenas, en un periodo insuficientemente tratado en la literatura especializada. Para ello hemos empleado información procedente de fuentes escasamente utilizadas en la historiografía agraria, especialmente el Catastro Agrícola. A partir del mismo, hemos construido y procesado una base de datos de más de medio millón de registros provenientes de los tres primeros catastros agrícolas, realizados en 1832, 1837 y 1852. Nuestros resultados indican que la distribución tanto del ingreso agrícola como de la tierra en Chiloé era muy igualitaria, lo que constituye una excepción dentro de todas las provincias chilenas c.1830-1855. Esta excepcionalidad de la sociedad rural de Chiloé se explica principalmente por políticas aplicadas desde fines del periodo colonial, en particular la entrega de tierras a criollos e indígenas, así como por un proceso de partición y venta de tierras realizado poco después de la independencia, un fenómeno único en Chile.
\end{abstract}

PALABRAS CLAVE: Chiloé, catastro, desigualdad, distribución de la tierra, Chile, siglo XIX.

\section{CHILOÉ, AN EGALITARIAN RURAL SOCIETY. AGRICULTURAL INCOME AND LAND DISTRIBUTION AFTER INDEPENDENCE}

\begin{abstract}
This article assesses both agricultural income distribution and land distribution in Chiloé during the 1830s-1850s, comparing both variables with the rest of the Chilean provinces at that time, in a rather unexplored period in Chile's agrarian historiography. We have used several untapped sources such as the Catastro (agricultural census). From this source, we have built and processed an over half a million records database for the first three agricultural censuses of 1832,1837 and 1852. Our results show a very egalitarian distribution of both agricultural income and land in Chiloé

\footnotetext{
Profesor Titular, Departamento de Economía, Universidad de Santiago de Chile. $\$ manuel.llorca@usach.cl.
}

b Profesor Asociado, Departamento de Economía, Universidad de Santiago de Chile. claudio.robles@usach.cl.

c Investigador Asociado, CIHEAP, FAE, Universidad de Santiago de Chile. juandonm@gmail.com.
\end{abstract}


c.1830-1855, an exceptionality within Chile. This uniqueness of Chiloé is mainly explained by policies applied from late colonial times, in particular the granting of land to creole and indigenous people, as well as by a process of land partition soon after independence, being both processes unique to Chile.

KEY WORDS: Chiloé, catastro, inequality, land distribution, Chile, nineteenth century.

\section{INTRODUCCIÓN}

Aunque la noción de una sociedad rural de poderosos latifundistas y sumisos inquilinos en Chile Central aún permanece en el imaginario nacional como la supuesta representación paradigmática del "campo chileno", la historiografía especializada ha puesto de relieve la pluralidad de sociedades rurales en nuestro país, cada una de las cuales tiene sus propios procesos formativos, trayectorias, estructuras, actores y, ciertamente, conflictos (Santana, 2006; Robles, 2009). En tal sentido, uno de los muchos tópicos respecto de los que se requiere mayor investigación es el de la estructura agraria, puesto que está directamente relacionada con la composición, en términos de clases, estratos y sectores, de las distintas sociedades rurales que existen en Chile.

En esa perspectiva, este artículo examina la estructura agraria de una sociedad rural insuficientemente estudiada por la historiografía económica y que, como veremos, destaca por su igualitaria distribución de la tierra y del ingreso agrícola: Chiloé. En efecto, este rasgo distintivo de la sociedad rural del archipiélago puede apreciarse a través del análisis de la información contenida en una fuente documental extraordinaria, como es el denominado Catastro Agrícola. En 1831, tan sólo cinco años después de la anexión de Chiloé al territorio chileno, el primer gobierno de José Joaquín Prieto decidió introducir un nuevo impuesto agrícola en Chile, denominado Catastro, que rigió hasta 1861 (Eyzaguirre Escobar, 19771978). Dicha gabela gravaba con un $3 \%$ el ingreso anual de mercado de todos los fundos de Chile cuya renta fuese superior a los $\$ 25$ anuales (i.e. los ingresos por ventas de productos en el mercado, Álvarez, 2014). Para determinar quiénes debían pagar dicho gravamen, los respectivos gobiernos chilenos realizaron tres censos agrícolas o catastros (término del que proviene el nombre de este impuesto), para la totalidad de la República (en el caso del primero) o buena parte de ella (los siguientes dos). Los referidos censos se condujeron en 1832 $1834,1837-1838$ y $1852^{1}$, y su realización fue un hecho sin precedentes, pues nunca antes ninguna república latinoamericana había emprendido semejante iniciativa.

La cobertura de los sucesivos catastros fue desigual. El primer censo agrícola fue universal, porque la totalidad de las casi 22 mil propiedades rurales de Chile en ese entonces fue catastrada (Tabla 1). Chile se convirtió así en un pionero en las estadísticas agrícolas de cualquier país de la región. En los censos posteriores, sin embargo, sólo se catastraron aquellas propiedades que se estimase generaban más de $\$ 25$ por año para el mercado, por lo que las propiedades censadas en el segundo catastro bajaron a poco menos de 10 mil. En cambio, en el tercer catastro se incluyeron más de 33 mil predios, como resultado de dos procesos; por un lado, una subdivisión predial y, por otro, el aumento del precio de los productos agrícolas, que, a su vez, tuvo como consecuencia un incremento en el ingreso anual de mercado de los predios, temas que han sido tratados en otros trabajos.

En cualquier caso, para los dos primeros censos, las variables que se ingresaron para cada uno de estos predios fueron: provincia, parroquia, partido, nombre del predio, nombre del propietario, cuadras del predio (superficie), plantas de viña, ganado mayor, ganado menor y renta anual (ingreso). En el Apéndice se incluyen varios ejemplos para algunas propiedades de Chiloé y de Colchagua, con el propósito de ilustrar al lector sobre la naturaleza de la principal fuente primaria utilizada (Tabla A.1). Lamentablemente, 
en el último censo no se registró información sobre superficie, plantas de viña, ganado mayor y ganado menor. Para que el lector pueda apreciar las diferencias entre ambos tipos de censos, otro ejemplo de cómo se presentaba la información para ese último catastro de 1852 fue también incluido en dicho Apéndice (Tabla A.2), esta vez sólo para el caso chilote. A pesar de la pérdida de datos que habrá constatado el lector al comparar las dos tablas del Apéndice, la riqueza de la información contenida en los catastros agrícolas es extraordinaria. De hecho, ella nos permite estimar con precisión indicadores clásicos para medir desigualdad, tales como coeficientes Gini de distribución del ingreso agrícola de mercado, así como de la tierra, además de otras variables para medir desigualdad (e.g. porcentaje de familias sin acceso a la tierra), en tiempos en que Chile era eminentemente una sociedad rural y, por tanto, la desigualdad en el sector agrario representa una muy buena aproximación a la desigualdad en el conjunto del país.

Sin embargo, muy pocos historiadores (chilenos o extranjeros) han podido aprovechar la riqueza de información de los catastros agrícolas para realizar trabajos sustentados en análisis de grandes volúmenes de datos cuantitativos, como los que sustentan este artículo. Una de las razones fue la inexistencia de equipos electrónicos que permiten recopilar datos in situ en los archivos (e.g. laptops), así como de procesadores de bases de datos de fácil acceso y manejo (e.g. Excel, Stata), cuestión que se subsanó solo en las últimas décadas. Creemos firmemente que somos el primer equipo de investigadores en registrar en bases de datos electrónicas los tres catastros realizados en Chile entre 1832 y $1852^{2}$.

El análisis de la base de datos construida con la información disponible en los referidos catastros pone de manifiesto la peculiaridad de Chiloé. En este gran archipiélago, como en ninguna otra provincia chilena de la época, la distribución tanto del ingreso agrícola de mercado como la de la tierra era notablemente igualitaria, un rasgo muy distinto a lo que ocurría en el resto de Chile (salvo quizás

2 Otros autores han hecho uso de esta fuente, pero para fines muy específicos. Por ejemplo, trabajos conocidos que emplearon información fragmentaria proveniente de los catastros son los de Bauer, 1970; Eyzaguirre Escobar, en la provincia de Valdivia, cuya población era, no obstante, poco significativa). En este artículo, por tanto, estableceremos con evidencia empírica y explicaremos la singularidad de la estructura agraria de Chiloé, realizando así un aporte significativo a la historia económica y agraria del sur chileno en un periodo (c.1830-1860) poco cubierto por la historiografía económica. En efecto, la mayor parte de la historiografía sobre Chiloé se ha concentrado en el periodo colonial y el siglo XX, así como en temas lejanos a la actividad económica, descuidando de buena manera las primeras décadas de desempeño económico del Chile republicano (León, 2004; Morales, 2014, p. 41; León, 2015, pp. 12-13). Así, un autorizado especialista en la historia de la isla sostuvo recientemente que la vida económica del archipiélago durante el siglo XIX permanece como 'una de las características menos conocidas de este territorio' (León, 2015, p. 134).

Nuestras fuentes primarias de información para los dos primeros catastros son el Fondo Dirección General de Estadísticas, Tomos I-VII, Registro de Predios Rústicos, disponible en el Archivo Histórico Nacional de Chile (ANCH-FDGE de aquí en adelante). Para el tercer catastro hemos consultado versiones impresas del mismo, ambas disponibles en la Biblioteca Nacional de Chile ${ }^{3}$. En total ingresamos más de medio millón de registros. Adicionalmente hemos consultado diarios de viajeros y oficiales de gobierno (tanto para el periodo colonial tardío como para las primeras décadas de la independencia), anuarios estadísticos, diarios de la época, así como censos de la República y la bibliografía secundaria relevante al tema, entre otros textos. Cabe destacar también que, para nuestro periodo, los diarios de viajeros extranjeros son una fuente primordial de información, incluso quizás la más importante en términos cualitativos (León, 2015, p.18), siendo escritos principalmente por militares españoles, jesuitas, exploradores ingleses y cartógrafos de diversas nacionalidades (Urbina Carrasco, 2016a, p. 104).

El artículo se estructura de la siguiente manera: en la próxima sección se presenta una nota metodológica, para explicar con detalle las

1978-79; Mellafe, 1988; y más recientemente los de Álvarez Correa, 2014 y Lacoste, 2016.

Chile 1855 y Chile 1861 
características de la fuente principal y el tipo de información que contiene. A continuación, luego de procesar nuestras bases de datos y recopilar información de fuentes anexas, presentamos resultados generales de los catastros, con especial acento en la situación de Chiloé, incluyendo el número de fundos por provincia, población chilena y fundos por habitante, por ejemplo. En la sección siguiente reportamos algunos indicadores de desigualdad habituales en la bibliografía, principalmente el coeficiente Gini de ingreso agrícola y de la propiedad de la tierra, poniendo así de manifiesto la excepcionalidad de la provincia de Chiloé. Posteriormente, se explica por qué Chiloé tuvo tan buena distribución de la tierra y del ingreso agrícola de mercado en el periodo bajo estudio.

\section{Nota metodológica sobre el catastro}

Dada la complejidad y riqueza de la fuente principal en la que se basa este artículo, es pertinente incluir la siguiente nota metodológica. El impuesto del catastro, introducido en 1831, reemplazó a las alcabalas subastadas (un impuesto de $6 \%$ que se aplicaba a las ventas de productos agrícolas), impuestos de licores y el cabezón (Eyzaguirre Escobar, 1978-1979, p. 85; Salazar 1985, p. 119; Gay 2009, p. 66; Villalobos 2010, pp. 237238). Dicho cambio impositivo fue parte de una reforma tributaria mayor, que se realizó en el inicio del periodo portaliano. De acuerdo al Ministro de Finanzas de la época, esta modificación consideró la introducción del catastro como un ensayo para la posterior introducción de contribuciones directas en Chile (lo cual incluía a su vez la eliminación del diezmo), en un intento del gobierno por controlar mejor los ingresos potenciales provenientes del sector agrícola 4 . Una parte menos conocida del origen del catastro, pero resaltada por fuentes británicas contemporáneas (reportes consulares), es que fue introducido exclusivamente para pagar parte de los intereses y el capital del primer préstamo tomado por Chile en Londres, impago desde mediados de los años 1820, un tema que tratamos en un artículo anterior (Llorca-Jaña et al. 2017).

4 British Consular Reports from Chile, National Archives, UK (FO 16). Memoir of the Minister of Finance, Manuel Rengifo, 1835.
En términos operacionales, este nuevo impuesto era recaudado por funcionarios del Estado, a través de la Factoría General del Estanco (Chile, 1859, p. 307; Villalobos, 2010, p. 237), desechando así la alternativa de una recolección de gabelas realizada por privados (como en el caso de la alcabala), que se prestaba para abusos, arbitrariedades y consecuentes reclamos de parte de los contribuyentes ${ }^{5}$. Con este propósito, se estableció un importante nuevo aparato burocrático, también parte de reformas que pretendían mejorar la recolección de gravámenes. La nueva entidad era encabezada por la Junta Central del Catastro, integrada por 5 miembros (designados por el Intendente), a la que le correspondía nombrar una Junta Departamental, compuesta también de 5 miembros (el gobernador, el administrador del estanco y tres vecinos pudientes). A su vez, la Junta Departamental nombraba a una Junta Parroquial, integrada por el párroco local, un delegado del gobernador y tres vecinos adinerados, quienes finalmente eran responsables de recolectar la información primaria, la que luego debía ser validada por las dos juntas superiores y posteriormente enviada al Ministerio de Hacienda.

Nuestra principal fuente de información para recopilar la información recogida en estos censos agrícolas se encuentra en el Archivo Nacional de Chile. Para los dos primeros catastros hemos consultado el Fondo Dirección General de Estadísticas, Tomos I-VII, Registro de Predios Rústicos (ANCH-FDGE de aquí en adelante), mientras que para el tercer catastro hemos consultado una versión impresa disponible en la Biblioteca Nacional ${ }^{6}$. Ejemplos de la información disponible en estas dos fuentes se presentan en la Tabla A.1 y Tabla A.2 (Ver Apéndice). La primera de ellas contiene 15 lotes censados en el catastro de 1837-1838, para que el lector se haga una idea clara de la naturaleza de la información disponible. Los mismos fueron seleccionados de forma aleatoria para las provincias de Chiloé y Colchagua. El mismo número de predios fue incluido en la Tabla A.2, con datos del catastro de 1852, pero esta vez solo para Chiloé. Originalmente, el catastro

5 Ley del 23 de octubre de 1834, disponible en http://www. leychile.cl. Ver también Eyzaguirre Escobar, 1978-1979, p. 86. 6 Chile, 1855. 
equivalía a un $4 \%$ del valor del ingreso anual de mercado estimado de todos los fundos rústicos cuya producción estuviese avaluada en $\$ 25$, o más, por año7. Cabe destacar que se gravaba solo la producción que se destinaba al mercado, no aquella producida para autoconsumo. Sin embargo, debido a dificultades iniciales en su cobro, así como a reclamos de algunos propietarios por el alto porcentaje del nuevo impuesto, prontamente se optó por reducir dicha tasa a $3 \%^{8}$. La meta de los gobiernos de turno era recaudar alrededor de $\$ 100.000$ anuales a través de este gravamen, la que se alcanzó recién a principios de la década de $1850^{9}$. Dicha cifra no alcanzaba a cubrir la totalidad del pago del capital e intereses del empréstito en Londres, pero al menos pagaba parte importante de ello, además de tranquilizar a los tenedores de bonos.

\section{RESULTADOS GENERALES: CHILOÉ VERSUS CHILE}

El primer resultado del análisis de los censos agrícolas es que de los casi 22 mil predios agrícolas en que se distribuía Chile en 1832-1834, pagasen o no el catastro, un $35 \%$ de ellos se encontraba en Chiloé, por entonces una región periférica de la naciente república ${ }^{10}$. Si ya es extraordinario que

7 Chile, Principios elementales..., op.cit, p. 306. El impuesto debía ser pagado en octubre de cada año, ya sea por el propietario o por el agricultor arrendando la tierra.

8 Sesiones de los Cuerpos Legislativos. Cámara de Diputados. Acta de la sesión del 16 de octubre de 1834

9 El primer año de recaudación efectiva fue 1835.

10 Al respecto, cabe destacar que Chiloé, aunque lejano de "Chile" en 1830, era una región de antiguo poblamiento y ocupación permanente del territorio, mucho más estable no sólo que el Chile entre el Bío Bío y Osorno, sino también que de la región del Maule.

11 Las papas en particular fueron descritas por un visitante en los años 1770 como el "pan" del chilote. Beranger 1893 (1773), p. 39. Tal era la variedad cultivada de este tubérculo en el archipiélago que, haciendo gala de lo anterior, en 1856 el intendente de Chiloé hizo llegar a la Exposición Nacional de Agricultura unas 30 variedades de papas chilotas. Mensajero de la Agricultura, Tomo 1, p. 48

12 De acuerdo al redactor del Mensajero de la Agricultura, "la madera de este árbol es sin duda la más preciosa de Chile y tal vez de la América del Sur". Tomo 1, p. 48. En el mismo medio Vicuña Mackenna lo describió como "el árbol más magnífico que existe en Chile". Mensajero de la Agricultura, Tomo 2, p. 51. a algo más de uno cada tres predios agrícolas en Chile en ese entonces se encontraran en Chiloé, más lo es el que dicha provincia, todavía marginal en términos políticos y económicos, concentrara más del doble de los fundos que cualquier otra en Chile.

Antes de proseguir, vale la pena resaltar algunas características básicas de estos numerosos predios. En general, eran fundos pequeños, cercanos a la costa (Urbina Carrasco, 2011, p. 58), destinados a producir papas ${ }^{11}$ y otros productos para el autoconsumo (algo de trigo, cebada, quínoa, maíz y arvejas), cuyos propietarios combinaban la agricultura con una intensa actividad pesquera artesanal (Tabla 3). Así, la actividad agrícola estaba destinada principalmente a producir para el sustento del hogar, más que para comercializar productos en el mercado (León, 2015, pp. 40-41). En palabras de Vicente Pérez Rosales, quizás algo exageradas, pero haciendo una observación válida: "el chilote no es agricultor" (Pérez Rosales, 1859, p. 277), a pesar de su ruralidad. Otros productos importantes de la economía chilote eran la madera, sobre todo el alerce ${ }^{12}$, verdadera estrella del comercio exportador chilote ${ }^{13}$, la construcción de pequeñas embarcaciones (Rondizzoni, 1854), textiles (e.g. ponchos, mantas), jamones ${ }^{14}$ (Blanckley, 1834; Rondizzoni 1854; Urbina, 1987; Beranger, 1893),

13 Cuadra, 1866, pp. 272-273; García Huidobro, 1864, p. 445; Anónimo, 1851. Ver también Pérez Rosales, 1859 , p. 275; Urbina, 1983, pp. 73-74; León, 2015, pp. 134-135; Barros Arana, 2000, p. 129. Según Urbina Carrasco, 2011, p. 57, el corte y comercialización del alerce fue la principal actividad económica de Chiloé durante el periodo colonial y buena parte del siglo XIX. Asimismo, debido a la escasez de moneda en el archipiélago, tablas de alerce y papas eran usadas como moneda de cambio. García Huidobro, 1864, p. 453; Cuadra 1866 , p. 272 . Ver también Urbina Carrasco, 2011, pp. 6062.

14 Chiloé era uno de los pocos centros criadores de porcinos de Chile, siendo famoso por la calidad de sus jamones, según Pérez Rosales (1859, p. 278), éstos eran "los más afamados del mar del sur". Igual percepción tenía Benjamín Vicuña Mackenna, para quien "en Chiloé se fabrican muy buenos jamones para la marina". Mensajero de la Agricultura, Tomo 2, p. 48. Ver también Blanckley, 1834, p. 353; Poeppig, 1960, p. 144; Urbina Carrasco, 2016b, p. 134; Mujica et al 2017. En efecto, el ganado porcino era el más abundante del archipiélago, superando en cabezas a las cabras, vacas y ovejas. Beranger, 1893 (1773), pp. 41-42. Una consecuencia de esto fue que el consumo de carne de vacuno en Chiloé era de los más bajos de Chile. Rondizzoni, 1854. 
propios de una provincia con marcada fisonomía agraria, forestal y, desde luego, insular ${ }^{15}$. De hecho, de alguna manera esta estructura dominante de división de la tierra en pequeñas propiedades se debía en parte a la geografía de la isla, dominada por bosques espesos que dificultaban las actividades de siembra y $\operatorname{cosecha}^{16}$, aunque también por cuestiones políticas, como veremos más adelante. Finalmente, cabe destacar que durante la época colonial Chiloé vivía en un régimen casi autárquico, muy descuidado por el imperio español. Sólo unos pocos buques por año entraban desde el exterior a Chiloé, procedentes del Callao ${ }^{17}$, lo que también presupone una producción agrícola destinada al autoconsumo.

En cualquier caso, lo más extraordinario del gran número de fundos en el archipiélago es que Chiloé concentraba apenas un $4.3 \%$ de la población chilena en 1835 (el primer año para el que hay cifras confiables de población por región en Chile, ver Tabla 2). Dicho de otro modo, en este periodo (c.1832-1835), Chiloé registraba el ratio más bajo de personas por fundo (mientras más bajo este ratio, más igualitaria es la sociedad, ceteris paribus) a nivel provincial: 5.7 habitantes por fundo, en promedio, mientras que el total nacional era de 46 personas por propiedad rural. La provincia más cercana al ratio de Chiloé era Valdivia, pero con un lejano promedio de 21.3 habitantes por fundo, más de 3 veces superior al de Chiloé. Considerando que, en promedio, una familia chilena estaba constituida por unas 6 personas (Urizar, 1845, pp. 60, 69-70; Mellafe \& Salinas, 1988, p. 169; Salinas, 2004, p. 396), esto quiere decir que en Chiloé, circa 1835, prácticamente cada familia poseía su propio pedazo de tierra, algo único para Chile (y seguramente también excepcional dentro del continente, sino

15 Alrededor de un $75 \%$ de la población chilota era rural, de acuerdo a los censos de 1854 y 1865. Morales, 2014, p. 47.

16 El interior era muy boscoso en el archipiélago, lo que sin dudas dificultaba la explotación agrícola. Urbina, 1983, pp. 66-68; González de Agüero, 1791, pp. 83-84; Contreras et al. 1971, p. 13; Pérez Rosales, 1859, p. 276.

17 Entre 1598 y hasta gran parte del siglo XVIII solo un barco al año llegaba a Chiloé. A partir de 1770 se observa un aumento de este flujo, pero no más de diez barcos llegaban cada año, en un tema que necesita más investigación. Juan \& Ulloa, 1826; De Moraleda, 1790, p. 293; Urbina, 1983, del mundo), lo que, en suma, da cuenta de una sociedad rural bastante igualitaria. Corroborando lo anterior, Pérez Rosales, escribiendo a finales de los años 1850, no titubeó en aseverar que "todo chilote es propietario" (Pérez Rosales, 1859, p. 277), mientras que otro testigo en los años 1830 , el capitán británico Edward Blanckely (1834, p. 354), también observó que la tierra en Chiloé era "divided into numerous small possessions, so that each father of a family is the possessor of some portion of the soil", lo que fue constatado a mediados de los años 1860 por un autorizado analista de la agricultura chilena, Pedro Lucio Cuadra (Cuadra, 1866, p. 275) ${ }^{18}$. Cuarenta años más tarde, Recaredo Santos Tornero era de la misma opinión: "los terrenos están muy subdivididos, la mayor parte de los chilotes es propietaria de fundos" (Tornero, 1872, p. 385), e igual situación era constatada en una sección del Anuario Estadístico de 1874-1875 dedicada a Chiloé. Nuestra base de datos procedentes de los catastros agrícolas valida tales afirmaciones y complementa aseveraciones previas en relación al predominio de familias mononucleares en Chiloé en el siglo XIX ${ }^{19}$. Finalmente, cabe destacar que la superficie de la isla que era explotada era bastante menor en este periodo a lo que es hoy. Por ejemplo, pocos se aventuraban a la cordillera de Piuchué o a la vertiente occidental.

Para los periodos siguientes, 1837-1838 y 1852 , el total de fundos de Chile que no pagaban el catastro no fue registrado en los catastros. Dichos censos agrícolas sólo consignaron aquellos fundos que efectivamente pagaban el catastro, es decir, cuyo ingreso de mercado fuese igual o superior a \$25 por año. Dado el reducido tamaño de los fundos en Chiloé, y que los mismos no producían un volumen significativo para el mercado sino para

p. 85; Urbina Carrasco, 2013, p. 191.

18 Pedro L. Cuadra fue un activo miembro de la Sociedad Nacional de Agricultura y co-autor con Martin Droully de Ensayo sobre el estado económico de la agricultura en Chile, redactado para el Congreso Agrícola de París, Boletín de la Sociedad Nacional de Agricultura, Vol. IX, no. 14 (1878)

19 Según León (2015, p. 33), en el siglo XIX chilote, la mayor parte de la población vivía en propiedades pertenecientes a una sola familia. Barros Arana (2000, p. 127) ya había realizado una afirmación en esta línea: en Chiloé "está cada familia sola en su casa". 
Tabla 1. Número de fundos catastrados en Chile, por provincia, 1832-1852.

\begin{tabular}{|c|c|c|c|c|}
\hline \multirow{2}{*}{ Provincia } & \multirow{2}{*}{$\begin{array}{c}\text { Total Predios } \\
1832-4\end{array}$} & \multicolumn{3}{|c|}{ Predios que pagaban catastro } \\
\hline & & $1832-4$ & $1837-8$ & 1852 \\
\hline Norte & 1.840 & 763 & 1.110 & 2.712 \\
\hline Atacama & & & & 1.191 \\
\hline Coquimbo-Serena & 1.840 & 763 & 1.110 & 1.521 \\
\hline Valle Central & 9.276 & 3.723 & 6.328 & 19.313 \\
\hline Aconcagua & 1.383 & 900 & 1.156 & 2.074 \\
\hline Colchagua & 2.562 & 690 & 1.552 & 4.380 \\
\hline Maule & 3.271 & 958 & 1.538 & 4.387 \\
\hline Ñuble & & & & 3.176 \\
\hline Santiago & 2.060 & 1.175 & 1.709 & 2.788 \\
\hline Talca & & & 373 & 1.146 \\
\hline Valparaíso & & & & 1.362 \\
\hline Sur & 10.791 & 922 & 2.539 & 11.019 \\
\hline Arauco & & & & 939 \\
\hline Chiloé & 7.634 & 4 & 144 & 6.492 \\
\hline Concepción & 2.741 & 851 & 2.129 & 2.864 \\
\hline Valdivia & 416 & 67 & 266 & 724 \\
\hline TOTAL & 21.907 & 5.408 & 9.977 & 33.044 \\
\hline
\end{tabular}

Fuente: Elaboración propia en base a ANCH-FDGE, y Chile 1855.

el autoconsumo familiar, en 1832-1834 eran muy pocos los fundos chilotes que pagaban catastro, tal como ocurrió en 1837-1838. Esta situación cambió desde fines de los años 1840, cuando el precio de los principales productos agrícolas chilenos comenzó a aumentar, se introdujeron varias mejoras en la agricultura chilota (punto tratado en la última sección) y, como consecuencia, se desarrolló de mejor manera la potencialidad de la tierra agrícola chilota ${ }^{20}$. De este modo, muchos fundos en Chiloé comenzaron a generar ingresos de mercado por sobre $\$ 25$ al año, y fueron incluidos en el pago del catastro. Así las cosas, en 1852 casi 6.500 fundos chilotes pagaban el catastro (nuevamente, más que en cualquier otra

20 De acuerdo a León (2015, p. 161), 'debajo de la selva austral se ocultaba un suelo fecundo'. Vale decir, terrenos forestales podían ser reconvertidos fácilmente a agrícolas con muy buenos resultados.

21 Corroborando estas cifras, Pedro Lucio Cuadra también daba cuenta de más de 6.000 fundos en Chiloé en la década de 1860. Cuadra 1866, p. 275.

22 Cabe destacar que posterior a este catastro, en 1853, fueron segregadas de las provincias de Valdivia y Chiloé provincia chilena) ${ }^{21}$, lo que representaba un $20 \%$ de todos los fundos chilenos que cumplían con dicha obligación. Significativamente, en ese año, la población chilota seguía representando poco más del $4 \%$ del total nacional ${ }^{22}$.

En su conjunto, los catastros permiten analizar algunas características adicionales de los predios de Chiloé. Primero, su ingreso promedio (de los que pagaban catastro) era el más bajo de todas las provincias chilenas (Tabla 3 ), siendo apenas entre un $12 \%$ y un $19 \%$ del promedio nacional para los catastros de 18321834, 1837-1838 y 1852. En parte, esto se explica por el tamaño pequeño de los fundos (cosa que explicaremos posteriormente), pero

las tierras adyacentes al lago Llanquihue, convirtiendo dicho territorio en tierras de colonización. Adicionalmente, en 1861 fue creada la provincia de Llanquihue como tal, separándola más formalmente de Chiloé y Valdivia, por lo que la población de la antigua provincia de Chiloé se vio disminuida (en concreto, se restó a los habitantes del departamento de Carelmapu, el único que estaba en tierra firme, en Chile continental). León, 2015; Schwarzenberg \& Multizábal, 1926. 
Tabla 2. Población chilena por provincias, y participación porcentual en el total, 1835, 1843 y 1854 .

\begin{tabular}{|c|c|c|c|c|c|c|}
\hline Provincias & 1835 & 1843 & 1854 & 1835 & 1843 & 1854 \\
\hline Norte & 99.841 & 103.719 & 161.279 & $9,9 \%$ & $9,6 \%$ & $11,2 \%$ \\
\hline Coquimbo-Serena & 99.841 & 103.719 & 110.589 & $10 \%$ & $10 \%$ & $8 \%$ \\
\hline Atacama & & & 50.690 & $0 \%$ & $0 \%$ & $4 \%$ \\
\hline Valle Central & 739.439 & 743.951 & 1.029 .226 & $73,2 \%$ & $68,6 \%$ & $71,5 \%$ \\
\hline Aconcagua & 137.039 & 91.674 & 111.504 & $14 \%$ & $8 \%$ & $8 \%$ \\
\hline Colchagua & 167.518 & 150.284 & 192.704 & $17 \%$ & $14 \%$ & $13 \%$ \\
\hline Maule & 120.185 & 146.524 & 156.245 & $12 \%$ & $14 \%$ & $11 \%$ \\
\hline Nuble & & & 100.792 & $0 \%$ & $0 \%$ & $7 \%$ \\
\hline Santiago & 253.887 & 208.154 & 272.499 & $25 \%$ & $19 \%$ & $19 \%$ \\
\hline Talca & 60.810 & 71.381 & 79.439 & $6 \%$ & $7 \%$ & $6 \%$ \\
\hline Valparaíso & & 75.934 & 116.043 & $0 \%$ & $7 \%$ & $8 \%$ \\
\hline Sur & 171.054 & 236.131 & 248.615 & $16,9 \%$ & $21,8 \%$ & $17,3 \%$ \\
\hline Arauco & & & 43.466 & $0 \%$ & $0 \%$ & $3 \%$ \\
\hline Chiloé & 43.832 & 48.876 & 61.586 & $4,3 \%$ & $4,5 \%$ & $4,3 \%$ \\
\hline Concepción & 118.362 & 164.189 & 110.291 & $12 \%$ & $15 \%$ & $8 \%$ \\
\hline Llanquihue & & & 3.826 & $0 \%$ & $0 \%$ & $0 \%$ \\
\hline Magallanes & & & 153 & $0 \%$ & $0 \%$ & $0 \%$ \\
\hline Valdivia & 8.860 & 23.066 & 29.293 & $1 \%$ & $2 \%$ & $2 \%$ \\
\hline TOTALES & 1.010 .334 & 1.083 .801 & 1.439 .120 & $100 \%$ & $100 \%$ & $100 \%$ \\
\hline
\end{tabular}

Fuente: Censos de la República, 1835, 1843, y 1854.

también por el carácter atrasado del sector agrícola chilote, abajo referido, y que perduró al menos hasta mediados de los años 1830. Sin embargo, también se debe considerar que el clima excesivamente lluvioso era un obstáculo significativo a la productividad del sector. Segundo, el ingreso máximo de todos los fundos de Chiloé era también el ingreso máximo más bajo para cualquier provincia chilena de la época. Por ejemplo, en 1832-1834, el fundo que más rentaba en Chiloé generaba \$55 al año (para el mercado), pero el más rentable de Chile lo hacía por $\$ 14.000$. Vale decir, estamos hablando de diferencias abismales. Incluso en periodos posteriores, como 1838 o 1852, no existían fundos chilotes que generasen más de $\$ 400$ al año, nuevamente dando cuenta de una sociedad bastante igualitaria, donde prácticamente no existían grandes terratenientes de ingresos muy altos ("súper ricos"). En concordancia con lo anterior, el ya citado E. Blanckley (1834, p. 354), de visita en la zona, afirmaría que "there is not an individual in the island who has possession of land of the value of 1000 dollars [...] and only two or three are valued above 500 dollars", lo que coincidía con la categórica observación realizada por un visitante ruso al final de nuestro periodo de estudio: "sus habitantes, en general, no son ricos" 23 . Cabe destacar que dicha situación de pobreza era constatada ya desde el siglo XVII.

Tal como adelantamos, el bajo ingreso de mercado de los fundos chilotes se explica de buena manera por el reducido tamaño de los predios de la gran isla, así como por el volumen modesto del 
capital invertido en ellos, por ejemplo, el ganado. La Tabla 4 da cuenta de ello: la extensión promedio de todos los predios de Chiloé en 1832-1834 era de 5.7 hectáreas, mientras que para todo Chile alcanzaba 140 hectáreas en ese periodo. Respecto del stock ganadero, en 1832 el fundo chilote que más cabezas de ganado mayor tenía, (propiedad de José Andrés Oyarzún, natural de San Carlos), contabilizó sólo 80 cabezas, una cifra muy marginal si se compara con el resto de Chile. $\mathrm{Al}$ respecto, cabe observar que la ondulación del terreno, así como el clima de las islas no era propicio para la engorda de vacas o caballos, tal como observó Charles Darwin: "hay pocos pastos para los grandes cuadrúpedos, por lo cual los principales alimentos son los cerdos, las patatas y el pescado" 24 . Respecto del ganado menor, la situación no era muy distinta: había un fundo con 1.240 cabezas, la propiedad de Francisco Cheuquepil, natural de Quinchao, pero esto era bastante poco común. De hecho, en el catastro de 1832-1834 sólo 3 fundos registraron más de 100 cabezas de ganado menor. Finalmente, se debe destacar que prácticamente no se cosechaban viñas en Chiloé, lo que se explica por obvias razones climáticas. Un visitante en los años 1850 corroboraba lo antes dicho al señalar que: "there is neither wine nor spirit made to any extent in the province" 25 .

Siguiendo con nuestro análisis de resultados generales, en 1832-1834 Chiloé era la segunda provincia que menos ingresos agrícolas (de mercado) generaba, tan sólo un $0.8 \%$ del total nacional, sólo superando a Valdivia, que estaba escasamente poblada en aquella época (Tabla 5). De hecho, en términos per cápita, Chiloé poseía el menor ingreso agrícola medio (de lo que se

24 Yudilevich y Castro Le-Fort, 1995, p. 154. Gillis (1856, Vol. 1, pp. 74-75) también confirma lo dicho por Darwin: el ganado se multiplica a una tasa menor a la necesaria para mantener el consumo doméstico de Chiloé. Según De Moraleda (1790) y Beranger 1893 (1773), el reducido número de ganado se debía a la falta de pastizales y espacios abiertos. Al respecto, ver también Urbina, 1983, p. 71. Sin embargo, el pescado se encontraba disponible en grandes cantidades a un costo de extracción muy bajo, mientras que las papas se cultivaban de buena manera, lo que compensaba la falta de ganado, tanto en términos de consumo de proteínas y carbohidratos, como de calorías total. Sobre el alto consumo de pescados y mariscos dentro transaba en el mercado) de todo Chile, incluso por debajo de Valdivia. No en vano, escribiendo sobre el carácter de los chilotes en los años 1830, E. Blanckley (1834, p. 346), era de la idea que los chilotes "are satisfied with little, and only care for the present". Sin embargo, tener el menor ingreso agrícola per cápita de mercado de todo Chile no era impedimento para una buena repartición del ingreso y de la tierra, una conclusión que nos conduce a la próxima sección.

\section{Indicadores de desigualdad (e igualdad)}

Considerando los datos expuestos, el lector podrá haber anticipado que Chiloé pareciera ser una sociedad bastante más igualitaria que el resto de Chile. Sin embargo, esto debe ser demostrado con los indicadores usuales empleados por los especialistas en el tema. El primer indicador que emplearemos es el coeficiente $\mathrm{Gini}^{26}$ del ingreso agrícola de mercado. El único catastro para el cual tenemos información de todos los fundos de Chile, no sólo los que pagaban el catastro, es el de 1832-1834. Tomando el total de casos, el coeficiente Gini del ingreso agrícola de mercado para todo Chile alcanza un 0.897, un valor extremadamente alto. En cambio, el mismo indicador, esta vez para Chiloé solamente, alcanzó tan sólo un 0.129, muy cercano a una perfecta distribución del ingreso agrícola de mercado, a priori un resultado bastante contundente para sustentar la excepcionalidad de Chiloé en términos de una buena distribución del ingreso agrícola. Ya desde fines de la colonia (y en marcado contraste con la mayor parte del periodo colonial), un observador autorizado como José de Moraleda ${ }^{27}$, constató en 1790 que

de la dieta chilota, ver también Contreras et al. 1971 y Urbina 1983, pp. 71-72. Tan importante era el consumo de pescado, que era considerado, hasta bien entrado el siglo XIX, como la fuente principal de alimentos del chilote, junto a las papas. León, 2015, pp. 40-41.

25 Gillis, 1856, Vol. 1, p. 74.

26 El coeficiente de Gini es una medida de la desigualdad de cualquier variable, aunque normalmente se utiliza para medir la desigualdad en los ingresos dentro de un país.

27 Alférez de fragata, cartógrafo y primer piloto de la Real Armada española, quien navegó por casi 16 años en el Pacífico, designado por el Virrey del Perú para explorar las costas del sur de Chile. Schwarzenberg \& Multizábal, 1926. 
Tabla 3. Ingreso promedio e ingreso máximo de fundos que pagaban catastro, por provincias, 1832-1852.

\begin{tabular}{|c|c|c|c|c|c|c|}
\hline \multirow[b]{2}{*}{ Provincia } & \multicolumn{2}{|c|}{$1832-1834$} & \multicolumn{2}{|c|}{ 1837-1838 } & \multicolumn{2}{|c|}{1852} \\
\hline & $\begin{array}{l}\text { Ingreso } \\
\text { promedio de } \\
\text { los predios }\end{array}$ & $\begin{array}{l}\text { Ingreso } \\
\text { máximo de } \\
\text { los predios }\end{array}$ & $\begin{array}{l}\text { Ingreso } \\
\text { promedio de } \\
\text { los predios }\end{array}$ & $\begin{array}{l}\text { Ingreso } \\
\text { máximo de } \\
\text { los predios }\end{array}$ & $\begin{array}{l}\text { Ingreso } \\
\text { promedio de los } \\
\text { predios }\end{array}$ & $\begin{array}{c}\text { Ingreso } \\
\text { máximo de los } \\
\text { predios }\end{array}$ \\
\hline \multicolumn{7}{|l|}{ Norte } \\
\hline Atacama & & & & & 319 & 12.000 \\
\hline Coquimbo-Serena & 213 & 6.000 & 258 & 6.500 & 276 & 15.000 \\
\hline \multicolumn{7}{|l|}{ Valle Central } \\
\hline Aconcagua & 316 & 10.000 & 320 & 9.000 & 315 & 17.500 \\
\hline Colchagua & 262 & 6.000 & 267 & 12.000 & 253 & 17.900 \\
\hline Maule & 131 & 3.008 & 128 & 8.000 & 156 & 10.000 \\
\hline Ñuble & & & & & 116 & 5.670 \\
\hline Santiago & 605 & 14.000 & 567 & 16.000 & 777 & 89.000 \\
\hline Talca & & & 345 & 3.500 & 242 & 12.000 \\
\hline Valparaíso & & & & & 403 & 60.000 \\
\hline \multicolumn{7}{|l|}{ Sur } \\
\hline Arauco & & & & & 115 & 6.000 \\
\hline Chiloé & 34 & 55 & 37 & 325 & 42 & 400 \\
\hline Concepción & 83 & 600 & 152 & 8.000 & 124 & 3.009 \\
\hline Valdivia & 65 & 300 & 85 & 500 & 79 & 500 \\
\hline Total Chile & 284 & 14.000 & 277 & 16.000 & 225 & 89.000 \\
\hline
\end{tabular}

Fuente: Elaboración propia en base a ANCH-FDGE, y Chile 1855.

Tabla 4. Extensión de los predios en Chile y en Chiloé, 1832-1852.

\begin{tabular}{lcccc}
\hline \multicolumn{1}{c}{ Variable } & Total Predios & \multicolumn{3}{c}{ Predios que pagaban catastro } \\
& $1832-4$ & $1832-4$ & $1837-8$ & 1852 \\
\hline Todo Chile & & & & \\
\hline Ingreso mínimo de los predios, \$ & 0,1 & 25 & 25 & 25 \\
Extensión mínima, hectáreas & 0,1 & 0,4 & 0,4 & $\mathrm{ND}$ \\
Extensión promedio, hectáreas & 140 & 466 & 324,0 & $\mathrm{ND}$ \\
Extensión máxima, hectáreas & 56.520 & 56.520 & 64.370 & $\mathrm{ND}$ \\
& & & & \\
\hline Solo Chiloé & & & 25 & 25 \\
Ingreso mínimo de los predios, \$ & 0,1 & 25 & 1,6 & $\mathrm{ND}$ \\
Extensión mínima, hectáreas & 0,4 & 4,7 & 138 & $\mathrm{ND}$ \\
Extensión promedio, hectáreas & 5,7 & 96 & 2.198 & $\mathrm{ND}$ \\
Extensión máxima, hectáreas & 314 & 314 & & \\
\hline
\end{tabular}

Fuente: Elaboración propia en base a ANCH-FDGE, y Chile 1855. 


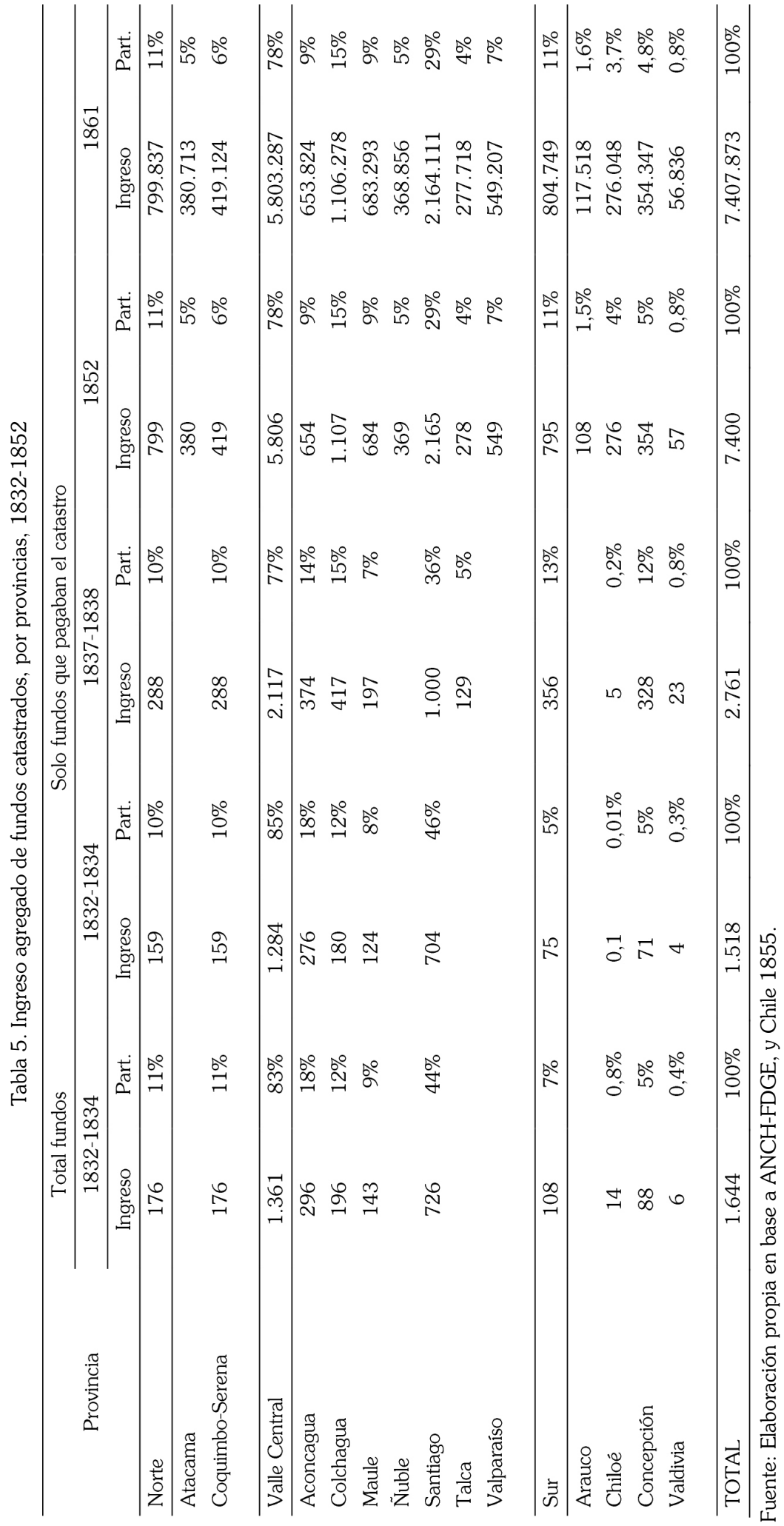

"la única diferencia [...] entre el más rico y el más pobre habitante de Chiloé, era que el primero acopiaba más trigo, cebada y papas que el segundo, para no padecer indigencia en los últimos meses del año ... y que era muy raro encontrar un hombre que poseyese un caudal de cien pesos en dinero" (Schwarzenberg \& Multizábal, 1926). De este modo, le evidencia cualitativa proporcionada por viajeros respalda nuestra evidencia cuantitativa.

La Fig. 1, que presenta la distribución del ingreso agrícola de mercado de los propietarios de fundos que pagaban el catastro en cada provincia, es también elocuente: Chiloé tiene el coeficiente Gini más bajo de ingreso agrícola de todas las provincias chilenas en los tres primeros catastros de 1832-1834, $1837-1838$ y 1852 , alcanzando coeficientes de $0.17,0.27$ y 0.29 , respectivamente. El total para Chile en estos tres periodos era de 0.75 , 0.75 y 0.79 , respectivamente. En consecuencia, la distribución del ingreso agrícola de mercado en Chiloé (entre los que pagaban catastro) era muchísimo mejor que en el resto de Chile y bastante igualitaria en términos absolutos. A fin de proporcionar perspectiva para que el lector pueda ponderar estos resultados, es pertinente señalar que las sociedades más igualitarias en el presente, a nivel mundial, tienen coeficientes Gini de distribución del ingreso (total) cercanos a $0.25^{28}$.

28 Ver, por ejemplo, datos recientes de Naciones Unidas, disponibles en http://hdr.undp.org/es/content/ income-gini-coefficient (accesado el 21 de noviembre de 2016). 


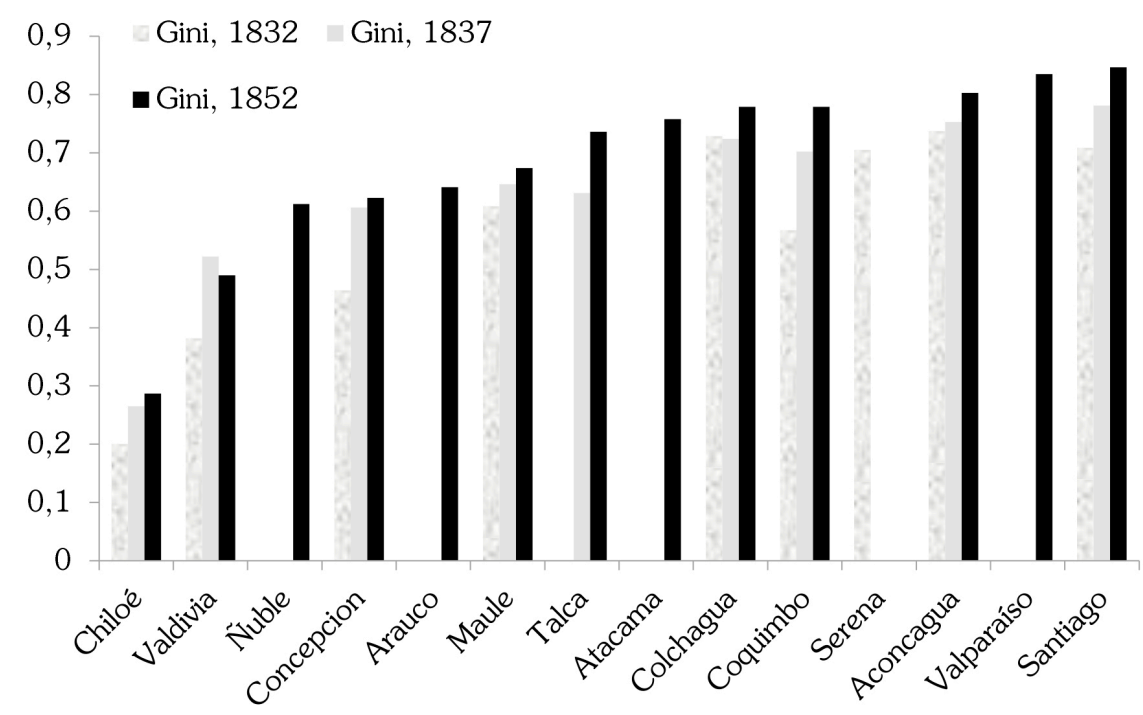

Fig. 1. Coeficientes Gini de ingreso agrícola de mercado por provincias, para aquellos predios que pagaban el catastro, 1832, 1837 y 1852.

Fuente: Elaboración propia en base a ANCH-FDGE, y Chile 1855.

Tabla 6. Participación en el ingreso agrícola total de los súper ricos, 1832 y 1852.

\begin{tabular}{lcccc}
\hline & Indicador & $1832-1834$ (todos los predios) & 1852 (solo predios que pagaban el catastro) \\
\cline { 2 - 5 } & Chiloé & Chile (Total) & Chiloé & Chile (Total) \\
\hline $1 \%$ más rico & $9 \%$ & $46 \%$ & $5 \%$ & $37 \%$ \\
$5 \%$ más rico & $24 \%$ & $76 \%$ & $16 \%$ & $65 \%$ \\
$20 \%$ más rico & $52 \%$ & $93 \%$ & $40 \%$ & $82 \%$ \\
$20 \%$ más pobre & $4 \%$ & $0,2 \%$ & $11,0 \%$ & $2,1 \%$ \\
20 más rico / 20 más pobre & 13,0 & 465,0 & 3,6 & 39,0 \\
\hline
\end{tabular}

Fuente: Elaboración propia en base a ANCH-FDGE, y Chile 1855.

Un segundo indicador de la distribución del ingreso de cualquier variable es considerar la proporción del total que corresponde al $1 \%$, el $5 \%$ y el $20 \%$ más rico de la población bajo estudio $^{29}$. En nuestro caso en particular, podemos realizar dicho ejercicio con el ingreso agrícola de mercado. Para ello hemos seleccionado solamente los catastros de 1832-1834 y el de 1852, por diversas razones. Primero, en el caso del primer catastro, el mismo concentra la totalidad de los fundos de la República (pagasen o no el catastro), lo que nos permite trabajar con el universo entero, ideal en cualquier muestreo. Hemos ignorado el catastro de 1837-1838 porque el mismo sólo contabilizó los fundos que pagaban el catastro, y en dicho censo los fundos de Chiloé que pagaban el impuesto fueron muy pocos $^{30}$. Optamos, en cambio, por incluir el
29 Para metodologías sobre cómo medir desigualdad en economías pre-industriales, ver Milanovic et al. 2007; Prados, 2007; Williamson, 2010; Bértola et al. 2010a, 2010b; Willebald, 2015.

30 Es probable que el reducido número de fundos chilotes que pagaban catastro en 1832-1834 y 1837-1838 pueda deberse a una decisión política, tomada en Santiago, de no gravar a los habitantes de Chiloé con esta nueva gabela, sobre todo considerando el aislamiento de este territorio y el hecho de haber sido anexado a Chile recién en 1826. 
de 1852 pues ya tenemos en ese catastro una buena muestra para el total de fundos de Chile y de Chiloé (cerca del $80 \%$ del universo total), y en realidad en dicho catastro se incluyó buena parte de los fundos de la República, toda vez que alrededor de dicho periodo hubo un pequeño boom en el sector, producto de exportaciones a los mercados de California y Australia, lo que hizo subir el precio de los principales productos de exportación del agro chileno y, por tanto, el ingreso de mercado de los fundos.

Nuevamente, el caso chilote destaca por su notable distribución del ingreso agrícola de mercado (Tabla 6). Si en 1832-1834, el 1\% más rico de los propietarios de predios rurales en Chile concentraba un $46 \%$ del total de todos los ingresos agrícolas de Chile, en el caso de Chiloé este ratio alcanzaba sólo al 9\%. A su vez, el 20\% más rico en ese censo captaba 465 veces más ingreso que el $20 \%$ más pobre en Chile, pero en el caso de Chiloé "sólo" 13 veces más. Para 1852 los indicadores para Chile mejoran, pero sigue habiendo bastante desigualdad en la distribución del ingreso agrícola de mercado así medida. Por su parte, la distribución del ingreso agrícola en Chiloé también mejora: el 1\% más rico de ahora percibe solo el $5 \%$ del total del ingreso (para Chile entero el ratio es de $37 \%$ ). Siguiendo con Chiloé, el $20 \%$ más rico acumula en 1852 sólo 3.6 veces más ingreso que el $20 \%$ más pobre de todos los propietarios, sin duda un muy buen indicador de distribución del ingreso agrícola de mercado.

Un tercer indicador que debemos analizar es el coeficiente Gini de la distribución de la propiedad de la tierra. Este ratio podemos calcularlo para los dos primeros catastros, pues en el tercero y cuarto esta información no está disponible. Sin embargo, como el segundo catastro está disponible sólo para aquellos que pagaban el catastro, y pocos fundos lo hicieron en 1837-1838 en Chiloé, nos concentraremos en el primer censo agrícola, considerando la totalidad de los fundos de la República, pagasen o no el catastro. De este modo, para 1832-1834 encontramos un coeficiente Gini de distribución

31 Parece ser el gobernador de la isla Lemuy. Darwin menciona al gobernador Gómez en 1834. Yudilevich \& de la tierra para todo Chile de 0.92, el cual es extraordinariamente alto. El ratio equivalente para Chiloé es sólo 0.39, muy por debajo del total nacional, lo que no es de extrañar considerando que el propietario que más tierra concentraba en Chiloé, Manuel Gómez ${ }^{31}$ de la isla Lemuy (la tercera más grande del archipiélago de Chiloé), poseía un fundo de "sólo" 314 hectáreas. En total, había solo 5 propietarios que concentraban más de 50 hectáreas entre todos sus fundos. Complementando esta información, cabe mencionar que sólo un $10 \%$ de los habitantes de Chiloé poseía más de un fundo en 18321834. No tenemos información de distribución de la tierra para los otros periodos para el total de Chile, pero podemos suponer que no varió considerablemente entre el inicio y el final del periodo que cubre nuestro estudio, no al menos como para cambiar un hecho innegable: Chiloé tenía la distribución de la tierra más igualitaria de todo Chile.

Finalmente, un cuarto indicador de desigualdad ampliamente usado es el porcentaje de familias que no poseen tierra en el sector rural. Para el total de Chile rural, en 1832-1834 estimamos que un $86 \%$ de las familias no eran propietarias de terreno alguno, un porcentaje sumamente alto y que permite corroborar que la distribución de la tierra en Chile era muy desigual. Para el caso de Chiloé, combinando los datos del censo de 1835 con los del catastro de 1832-1834 (periodos bastante cercanos), estimamos que sólo un $6 \%$ de la población, como máximo, podría no haber sido propietaria de algún lote de tierra, nuevamente confirmando la buena distribución de la tierra. En esta misma línea, un historiador de la isla confirma esa noción, afirmando que: "las ideas comunistas, tan divulgadas en toda suerte de escritos, en Chiloé no prenden o prenden con dificultad por la razón de que aquí el número de propietarios es muy subido. En algunas regiones del archipiélago casi no hay quien no sea dueño de una, dos, tres, cuatro o más cuadras de terreno, de una casita, de una yunta de bueyes y algunas ovejas a lo menos" (Barrientos, 2013, p. 197). 
¿Cómo se explica la excepcionalidad de Chiloé?

En la sección precedente hemos aportado sólida evidencia cuantitativa (pero complementada con observaciones de viajeros de la época), que revela la existencia de una sociedad rural muy igualitaria en Chiloé; de hecho, la más igualitaria de Chile. En esta sección consideramos los principales desarrollos que tuvieron lugar en Chiloé en la época colonial y examinamos cómo impactaron en la distribución de la tierra y del ingreso agrícola. Posteriormente haremos similar análisis para el periodo posterior a 1826, año en que finalmente Chiloé fue anexado a territorio chileno. Antes de proseguir cabe destacar que durante las últimas décadas del periodo colonial Chiloé dependió directamente del Virreinato del Perú (desde 1768), no así de la Capitanía General de Chile, descansado en buena medida del real situado para su subsistencia (Urbina Carrasco, 2013, p. 189).

Por su ubicación estratégica en el Pacífico sur, las autoridades españolas dieron gran importancia al control del archipiélago de Chiloé, cuestión que se acrecentó con el mayor uso que se comenzó a hacer de las rutas del Cabo de Hornos y del Estrecho de Magallanes (Urbina 1983; Guarda, 2002; León, 2015, pp. 134-136). La creciente presencia inglesa $y$, en particular, la holandesa en las aguas del Pacífico sur, determinó que la corona española considerara como vital tener dominio absoluto de Chiloé. De esta manera, el archipiélago fue rápidamente conquistado y repartido como botín entre conquistadores encomenderos (Guarda, 2002), marginando a la población indígena, tal como ocurrió en el resto de Chile y América. Así, desde inicios de la colonia, en Chiloé "los indios no podían poseer tierra" (Yudilevich \& Castro LeFort, 1995, p. 159), y de hecho el archipiélago estaba dividido en tan sólo cien encomiendas, que habían sido entregadas a un pequeño grupo

32 Sobre las encomiendas y las mercedes de tierras asociadas a las mismas, ver Poeppig, 1960, p. 189; Molina \& Correa, 1996; Molina, 1987

33 Este punto es corroborado por Urbina Carrasco, 2016b, p. 134.

34 Ver también Molina, 1987.

35 Paradojalmente, la encomienda se abolió en Chiloé primero que en el resto de Chile. Contreras et al. 1971, p. 9. de españoles ${ }^{32}$. De visita en la provincia en 1782 , José de Moraleda (1790, p. 794) observó que "hay muchos vecinos que no tienen un palmo de terreno propio fuera de su casa, al paso que otros poseen leguas enteras que conservan siempre eriales". Según el explorador y botánico germano Eduard Poeppig (1960, p. 189), quien visitó el país en los años 1820, "si Chile no fue tratado con mucho afecto por el gobierno español, podría decirse que éste tuvo por Chiloé los sentimientos de una madrastra [...] todo el grupo de islas estaba dividido en cien encomiendas [...] Los pobres indígenas eran siervos de los encomenderos, y eran tratados en forma más severa que en Perú"33. Vale decir, no necesitamos de mayores antecedentes para concluir que había una muy mala distribución de la tierra entre los habitantes del archipiélago en el periodo colonial ${ }^{34}$. Finalmente, respecto de la demografía del archipiélago, cabe destacar que durante la mayor parte del periodo colonial, incluido el siglo XVIII, Chiloé presenta dos características distintivas si se le compara con el resto de Chile. Por un lado, un aumento notable de la población sujeta a encomienda (incluso cuando dicho sistema de trabajo decaía en el resto de Chile) ${ }^{35}$, y por otro lado un alto componente de población indígena combinado con poco mestizaje (Contreras et al. 1971) ${ }^{36}$. De acuerdo a Urbina Carrasco (2013, p. 187), circa 1826 la mitad de la población chilota era indígena.

Esta situación comenzó a cambiar en parte durante fines del periodo colonial, en particular desde que el régimen de encomiendas fue abolido en Chiloé en 1783 (1791 en Chile), luego de una larga batalla política ${ }^{37}$. Incluso antes, con la expulsión de los jesuitas (1767), quienes poseían muchas propiedades en Chiloé, muchas de sus haciendas fueron vendidas $\mathrm{O}$ arrendadas a particulares, o bien entregadas a los indígenas (Donoso \& Velasco, 1928, p. 238). A partir de este momento, criollos, mestizos e indios, antes marginados de

36 Que haya existido poco mestizaje es fundamental para explicar el gran número de propietarios de tierra que se observa desde los años 1830, toda vez que la partición de tierra de fines de la colonia, por mandato real, así como la Ley Freire, benefició en particular a los huilliche.

37 Al respecto, ver Urbina, 1983, pp. 138-140; Urbina Carrasco, 2013, p. 190. 
la propiedad de la tierra, comenzaron a adquirir sus propias parcelas, iniciando un interesante proceso de subdivisión del suelo (Barrientos, 1932, p. 57). Los primeros grandes beneficiados fueron los habitantes españoles (no encomenderos) del archipiélago, que comenzaron a adquirir lotes tan pequeños como de dos cuadras de tierra, lo que fue combinado con la regularización de la propiedad de la tierra ya ocupada. ${ }^{38}$ Adicionalmente, en los últimos años del periodo colonial, en especial entre 1804 y 1825, la corona otorgó títulos de propiedad a las parcialidades huilliche en la costa de los payos en el Partido de Castro, reconociendo de algún modo la propiedad ancestral huilliche, en particular de los llamados "potreros realengos", aunque a cambio de un modesto por parte de los caciques (Torrealba, 1917; Molina, 1987; Molina \& Correa, 1996), marcando un hito en la historia de Chile, al menos en lo que guarda relación con la restitución de tierras a pueblos originarios, de la que otros pueblos fueron, y han sido, marginados.

Luego de la independencia, las propiedades españolas e indígenas quedaron salvaguardadas por el Tratado de Tantauco (1826). Hubo un reconocimiento del Estado chileno de las tierras vendidas en el proceso antedicho gracias a indicaciones de Antonio de Quintanilla, la última autoridad realista del archipiélago (Donoso \& Velasco, 1928, p. 248; Molina \& Correa, 1996, p. 16; Urbina Carrasco 2016b, p. 167). Sabemos además que sólo se aplicó en Chiloé, sobre todo desde 1829, una importante ley aprobada en Chile en 1823, la "Ley Freire", que respetaba la propiedad de la tierra de los nativos y estimulaba la división de tierra, en particular la creación de pequeños loteos de entre una y diez cuadras (Blanckley, 1834, pp. 354-355; Molina, 1987; Molina \& Correa, 1996) ${ }^{39}$. Dicho cuerpo legal permitía que tierra originalmente de indígenas se declarase propiedad de los mismos a perpetuidad y que aquella tierra estatal que a la fecha estuviese

38 En 1821, en particular, se dispuso la venta de tierras a ocupantes españoles, en lotes de 2 cuadras de terreno, y se regularizó además la ocupación de tierra sin título real. Molina \& Correa, 1996, pp. 13-14. Para algunos ejemplos concretos, ver Donoso y Velasco 1928, p. 241.

39 La ley de 1823 se cumplió estrictamente en Chiloé, al menos respecto del tamaño máximo de 10 cuadras de los lotes que podían venderse. Donoso \& Velasco, 1928, p. 251. Ver también Urbina Carrasco, 2016b, p. 167. sin dueño definido, alrededor de un $20 \%$ del total, fuese vendida en subastas públicas y en pequeños lotes, tan pequeños como aquellos con una superficie total de apenas 1.5 hectáreas, con el propósito principal de "proporcionar a muchos el que puedan ser propietarios" 40 . Tan potente y efectivo fue este mecanismo (que involucró a más de dos mil hogares huilliche), que, según Charles Darwin, de visita en el archipiélago poco después de puesta en marcha esta iniciativa, si un chilote no tenía tierra esto se debía "a su propia negligencia", pues adquirir tierra en Chiloé era visto como empresa muy sencilla. Bastaba con pagar por la mensura del terreno (2 chelines solamente por cuadra), tasar la tierra, y luego subastarla. Una vez tasada la tierra, la misma se debía ofrecer en subasta tres veces y si nadie hacía una oferta mejor, pasaba a ser propietario el primer postulante por el precio fijado inicialmente por el agrimensor (Darwin 1988, 238) ${ }^{41}$. Tales facilidades explican de buena manera los coeficientes de distribución de la propiedad de la tierra presentados en la sección precedente, toda vez que dicho cuerpo legal fue virtualmente ignorado en el resto de Chile. Así, como señalaran dos expertos en el tema, "la distribución de la propiedad territorial en Chiloé obedeció a circunstancias especialísimas, distintas a las que concurrieron en las otras provincias australes del país" (Donoso \& Velasco, 1928, p. 234), observación que también se aplica para el resto de Chile.

Otra transformación que incidió en una mejor distribución de la tierra y el ingreso agrícola fue la introducción de algunas innovaciones técnicas en la agricultura de Chiloé en la década de 1830. Durante el periodo colonial y en las primeras décadas después de la independencia, la agricultura chilota mantuvo su carácter atrasado, una situación bien descrita por Urbina (1983, pp. 69-70). Así, por ejemplo, tan tarde como en la década de 1770, un observador afirmaba que

40 Cita textual del artículo 5 de la "Ley Freire". Citado en Donoso \& Velasco, 1928, p. 250. Para ello, el gobierno chilote empleó topógrafos, encargados de medir y marcar los terrenos de la isla, un proceso de medición de terrenos que se realizó principalmente entre 1829 y 1837 , a cargo del agrimensor Silvestre Martínez. Molina \& Correa, 1996, pp. 16-17; Donoso \& Velasco, 1928, pp. 250-251.

41 Traducción propia. 
"no se conoce el uso del arado [de hierro], ni es fácil introducirlo", agregando que "el modo de laborear la tierra ... es penoso" (Beranger, 1893 (1773), pp. 38-39), situación que persistía en 1800 , cuando se seguía constatando la ausencia de instrumentos de labranza en Chiloé tan básicos como las yuntas de bueyes y el arado de fierro. Este manifiesto atraso en las técnicas agrícolas comenzó a cambiar a partir de los años 1830 y 1840, bajo la administración del gobernador Juan Agustín Vives, cuando nuevos implementos fueron introducidos con éxito en la isla, esto es, el arado $y$ herramientas de hierro ${ }^{10}$, aumentando así de manera significativa el ingreso de los fundos y el interés de potenciales agricultores para poseer sus propios lotes, empujándolos a participar en las subastas ya mencionadas. Además, una gran cantidad de molinos pequeños proliferaron en el archipiélago, llegando a tener la mayor cantidad de molinos que cualquier otra provincia de Chile, aunque de tamaños muy reducidos (Pérez Rosales, 1859, p. 284; León, 2015, p. 163).

Junto con las primeras innovaciones en las técnicas agrícolas, la mejor distribución de la tierra y del ingreso agrícola en Chiloé se explica por el bajo precio relativo de la tierra en este archipiélago, dado su condición periférica en la dinámica de la economía chilena. Así, tan tarde como 1872, en palabras de Tornero, "la propiedad tiene en Chiloé un escasísimo valor" (Tornero 1872, p. 385). Esta era la consecuencia inevitable de su lejanía de los centros de consumo chilenos e internacionales, así como de la carencia de un buen puerto internacional, puesto que ni siquiera Chacao era muy propicio para la carga y el desembarco de navíos. Ante esas limitaciones, los potenciales excedentes agrícolas de Chiloé tenían que superar potentes obstáculos para poder ser exportados de manera rentable. En concreto, de acuerdo a estimaciones de Claudio Gay, en los años 1840 el precio de una cuadra de tierra en Chiloé era apenas un $12 \%$ del precio de una cuadra de tierra en Santiago (Gay, 2009, p. 69). Después de la independencia, una cuadra de tierra podía ser adquirida por el jornal que ganaba en una quincena un obrero no calificado

42 Según Barrientos (2003, p. 161), durante este periodo se introdujo con más fuerza el uso del arado de hierro, "varias prácticas rutinarias que se seguían en la agricultura sufrieron un cambio favorable, obteniéndose cosechas más en Santiago: apenas $\$ 2$ y ni siquiera era necesario pagar el precio de contado, pues también eran aceptados pagos diferidos (Donoso \& Velasco, 1928 , p. 242). Al respecto, cabe destacar que, a diferencia de otros sectores del sur de Chile, en Chiloé prácticamente no se entregaron tierras a colonizadores extranjeros.

Como en muchos aspectos de la realidad de Chiloé, el impacto de sus condiciones climáticas también se debe considerar para el singular carácter igualitario de su sociedad rural en el periodo considerado en este estudio. Así, en un clima que podía llegar a ser muy hostil por las abundantes lluvias y las bajas temperaturas, era poco probable sobrevivir sin tener techo, sin ser propietario. El mismo Darwin, de visita en la isla grande de Chiloé en 1834, observó que "en invierno el clima es detestable" y añadió que "por lo demás, no es mucho mejor en verano", porque "creo que hay pocos lugares en las regiones templadas del mundo donde llueva más" (Yudilevich \& Castro, Le-Fort 1995, p. 153). Finalmente, la demografía del archipiélago también guardaba relación con la distribución de la propiedad de la tierra. Entre circa 1790 y 1855 la población chilena prácticamente se triplicó (creció 200\%), mientras que la chilota creció apenas un 80\% (Gillis 1856, Vol. 1, p. 74), lo que bien podría explicarse debido a la menor proporción de mujeres respecto de hombres (al menos en 1826), algo único de Chiloé si se compara con el resto de Chile (Poeppig, 1960, p. 188). Un menor crecimiento demográfico, todo lo demás constante, finalmente debía generar una proporción menor de familias sin tierra.

\section{CONCLUSIONES}

En este artículo hemos analizado la distribución del ingreso agrícola y de la tierra en Chiloé entre c.1830 y los años 1850, comparando ambas variables con el resto de las provincias chilenas. Para ello hemos empleado información procedente de fuentes escasamente utilizadas en la historiografía agraria, especialmente el Catastro Agrícola. A partir del mismo, hemos construido

abundantes, productos más seleccionados y, por tanto, rendimientos más en armonía con las bondades del suelo". Ver también León (2015, pp. 161-163). 
y procesado una base de datos de más de medio millón de registros provenientes de los tres primeros catastros agrícolas, realizados en Chile en 1832, 1837 y 1852 . Nuestros resultados indican que la distribución tanto del ingreso agrícola como de la tierra en Chiloé era muy igualitaria, lo que constituye una excepción dentro de todas las provincias chilenas c.1830-1860.

Esta excepcionalidad chilota se explica principalmente por políticas aplicadas desde fines del periodo colonial, en particular la entrega de tierras a criollos $e$ indígenas, un reconocimiento muy importante de derechos de propiedad a indígenas antes marginados (salvaguardadas por el Tratado de Tantauco), así como por la aplicación eficiente de la Ley Freire, que generó un intenso proceso de partición y venta de tierras, en pequeños lotes, algo único en Chile para el periodo analizado. El bajo precio de la tierra en Chiloé, así como factores climáticos, también explican el sobresaliente igualitarismo de Chiloé. Con todo, independientemente del peso relativo de cada una de las causas de este excepcional desarrollo igualitario, testigos presenciales de la época coinciden en que esto se tradujo en buenos niveles de vida para la población. Según Poeppig (1960, p. 189), Chiloé destacaba en los años 1820 y 1830 por tener una elevada esperanza de vida dentro de Chile, así como por su alto nivel de instrucción escolar. De acuerdo a información proporcionada por Cuadra (1866), en 1854, al final de nuestro periodo de estudio, uno de cada tres hombres sabía leer en Chiloé, y una mujer por cada 27,5 mujeres (Cuadra, 1866, p. 275), cifras bastante positivas para la época, al menos en comparación con otras regiones de Chile $e^{43}$. En efecto, en 1859, Chiloé concentraba un $14 \%$ de todos los alumnos matriculados en Chile en instrucción, siendo sólo superada por Santiago (16\%), que concentraba un $19 \%$ de la población, comparado con sólo un 4\% de Chiloé (Anuario Estadístico, 1862-1863). Alrededor de este mismo periodo, el Capitán Blanckley, varias veces mencionado en este artículo, atestiguaba que en Chiloé "murders, robbery, or persons being in debt, are never heard of" (Blanckley, 1834, p. 356), llegando incluso a sostener que "the inhabitants are very cheerful, and

43 Por ejemplo, en Curicó, San Fernando y Osorno, 1 de cada 7.3, 6.7 y 8.7 hombres, respectivamente, sabía leer, muy appear the happiest race I ever beheld" (Blanckley 1834, p. 357). Sin dudas, la buena distribución de la tierra y del ingreso deben haber contribuido a explicar parte de esta felicidad.

Sin embargo, el proceso de partición de tierras en Chiloé no se prolongó en el tiempo. De acuerdo a información encontrada en los anuarios estadísticos de la república, en 1871 en Chiloé, excluido Ancud, había tan solo 4.668 fundos (Anuario Estadístico, 1870-1871_. El lector recordará que en el total de Chiloé en 1832-1834 había 7.634 lotes (Tabla 1). Si descontamos de esa cifra los de Ancud y los de Carelmapu (que en 1853 dejó de pertenecer a la provincia), llegamos a 6.645 fundos en 1832-1834, muy por encima de la cifra registrada en 1871 . Medio siglo después, en 1920, cifras reportadas también en el anuario dan cuenta de 7.017 propiedades agrícolas en Chiloé, un $7 \%$ del total de propiedades agrícolas de Chile (Anuario Estadístico, 1920), un guarismo bastante menor al 35\% registrado en 1832-1834. Vale decir, el proceso de partición de tierras en Chiloé sufrió un retroceso importante en la segunda mitad del siglo XIX e inicios del XX, un aspecto a ser tratado en investigaciones futuras.

\section{AGRADECIMIENTOS}

Este artículo fue financiado por el proyecto FONDECYT Regular no. 1150161 y por Proyectos Basales USA1498-LM032122. Los autores están muy agradecidos de Jaime Rosenblitt, Enzo Videla y Francisco Betancourt por su ayuda para identificar y conseguir fuentes de gran utilidad para la elaboración de este estudio.

\section{FUENTES PRIMARIAS}

Archivo Histórico Nacional de Chile. Registro de Predios Rústicos. Fondo Dirección General de Estadísticas, (tomos I-VII).

Beranger, C. (1893). Relación Jeográfica de la Provincia de Chiloé. Chile, Santiago: Imprenta Cervantes.

Blanckley, C. (1834). Account of the Island and Province of Chiloé. Journal of the Royal Geographical Society of London, 4, 344-361.

"Bosquejo jeográfico de la Provincia de Chiloé". (1851). Chile, por debajo de Chiloé. Memoria del Ministerio del Interior, 1865. 
Valparaíso: Imprenta de El Mercurio.

Censo. (1843). Censo general de la República de Chile, 1854. Chile, Santiago: Imprenta del Ferrocarril.

Chile. (1835). Repertorio chileno. Año de 1835. Chile, Santiago: Imprenta Araucana.

Chile. (1855). Estado que manifiesta la renta agrícola de los fundos rústicos para deducir el impuesto anual establecido, 1852. Chile, Valparaíso: Imprenta del Diario.

Chile. (1858). Censo general de la República de Chile, 1854.

Chile, Santiago: Imprenta del Ferrocarril.

Chile. (1866). Censo general de la República de Chile. 1865. Chile, Santiago: Imprenta Nacional.

Cuadra, P. L. (1866). Bosquejo jeográfico de Chiloé. Anales de la Universidad de Chile, (XVIII), 266-276.

Darwin, C. (1988). Charles Darwin the voyage of the Beagle. Nueva York: Mentor.

De Ulloa, A., \& Jorge, J. (1826). Noticias secretas de América. Inglaterra, Londres: Ediciones Turner.

De Moraleda, J. (1790). "Breve descripción de la provincia de Chiloé, su población, carácter de sus habitantes, producciones y comercio". Sagredo \& Leiva.

García Huidobro, C. (1864). Provincias meridionales de Chile. Su descripción según viaje hecho a ellas por don Carlos García Huidobro. Anales de la Universidad de Chile, (XXV), 439-489.

Gillis, J. M. (1856). The U.S. Naval Astronomical Expedition to the southern hemisphere, during the years 1849 '50-'51-'52. Chile: its geography.... Philadelphia.

Gónzalez de Agüero, P. P. ${ }^{44}$ (1791). Descripción historial de la Provincia y Archipiélago de Chiloé. España, Madrid: Imprenta de Don Benito Cano.

Pérez Rosales, V. (1859). Ensayo sobre Chile. Chile, Santiago: Imprenta del Ferrocarril.

Poeppig, E. (1960). Un testigo en la alborada de Chile, 18261829. Chile, Santiago: Zig-Zag.

Rondizzoni, J. (1854). Memoria que el intendente de Chiloé presenta al señor Ministro de Estado en el Departamento del Interior. Chile, Santiago: Imprenta sin Identificar.

Urizar, F. (1845). Estadística de la República de Chile: provincia del Maule. Chile, Santiago: Imprenta de los Tribunales.

\section{BIBLIOGRAFÍA}

Álvarez Correa, L. (2014). Catastro de propiedad en Chile: orígenes y evolución (Tesis Doctoral). Universidad de Barcelona, España.

Barrientos, P. (2013). Historia de Chiloé. Chile, Chiloé: Museo Regional de Ancud.

Barros Arana, D. (2000). Historia general de Chile, (VII). Chile, Santiago: Editorial Universitaria.

Bauer, A. (1970). Expansión económica en una sociedad tradicional: Chile central en el siglo XIX. Historia, 9(1), 137-235.

Bértola, L., Castelnovo, C., Rodríguez, J., \& Willebald, H. (2010a). Between the colonial heritage and the first globalization boom: on income inequality in the Southern Cone. Journal of Iberian and Latin American Economic History, 28-02.

Bértola, L., Prados de la Escosura, L., \& Williamson, J. G. (2010b). Latin American Inequality in the Long Run. Journal of Iberian and Latin American Economic History, 28-02.

Contreras Arias., \& Flores Marambio, E., Herrera Canales, I., Mazzei De Grazia, L, Rivera Navarro, A., Romero Sepúlveda, R. (1971). La Población y la economía de Chiloé durante la colonia (1567-1826). Chile, Universidad de Concepción: Instituto Central de Historia.

Donoso, R., \& Velasco, F. (1928). Historia de la constitución de la propiedad austral. Chile, Santiago: Imprenta Cervantes.

Eyzaguirre Escobar, J. (1977-1978). El primer censo agrícola chileno. Boletín de la Academia Chilena de la Historia, 90, 79-126.

Gay, C. (2009). Historia física y política de Chile. Agricultura (I). Santiago, Cámara Chilena de la Construcción.

Guarda, G. (2002). Los encomenderos de Chiloé. Chile, Santiago: Universidad Católica de Chile.

Lacoste, P. (2016). El pisco nació en Chile. Santiago: RIL Editores.

León, M. (2004). La vida económica de un mundo insular. Una caracterización de Chiloé en el siglo XIX. Revista Cultura de y desde Chiloé, 18, 55-78.

León, M. A. (2015). Chiloé en el siglo XIX: historia y vida cotidiana de un mundo insular. Chile, Valparaíso: Universitarias de Valparaíso.

Llorca-Jaña, M., Robles, C., Navarrete-Montalvo, J., Araya, R (2017). La agricultura y la élite agraria chilena a través de los catastros agrícolas, c.1830-1855. Revista Historia, 90, 597-639.

Mellafe, R,. \& Salinas, R. (1988). Sociedad y población rural en la formación de Chile actual: La Ligua, 1700-1850. 
Chile, Santiago: Universidad de Chile.

Milanovic, B., Lindert, P. H., \& Williamson, J. G. (2007). 'Measuring ancient inequality'. NBER Working Paper, (13550).

Molina, R. (1987). El pueblo huilliche de Chiloé: elementos para su historia. Santiago, Chiloé: OPDECH.

Molina, R., \& Correa, M. (1996). Territorios Huilliches de Chiloé. Santiago, Conadi.

Morales, D. (2014). El negocio de la madera: comerciantes y 'hacheros' de Chiloé, 1850-1875. Magallania, 42(2), 41-60.

Mujica, F., Lacoste, M., Lacoste, P., Castro, A., Muñoz, J. G., \& Martínez, F. (2017). Jamón de Chiloé: itinerario histórico de un producto típico de América del Sur (siglos XVIII y XIX), Cuadernos de Historia, 46(1), 55-82.

Norambuena, C., \& Ulianova, O. (comp.). (2000). Viajeros rusos al sur del mundo. Chile, Santiago: Dibam-Usach.

Prados de la Escosura, L. (2007). Inequality and Poverty in Latin America: A Long- Run Exploration. Hatton, T., O' Rourke, K., \& Taylor, A. (Eds.), The Compara tive Economic History, 291-315.

Robles-Ortiz, C. (2009). Agrarian Capitalism and Rural Labour: The Hacienda System in Central Chile, 1870-1920. Journal of Latin American Studies, 3(41), 493-526.

Salinas, R. (2004). Historia de la familia chilena. Rodríguez, P. (Ed.), La familia en Iberoamérica, 1550-1980. Colombia: Universidad Externado de Colombia.

Santana, R. (2006). Agricultura chilena en el siglo XX: contextos, actores y espacios agrícolas. Chile, Santiago: Centro de Estudios Regionales y Locales/Centro de Investigaciones Diego Barros Arana.

Schwarzenberg, J., \& Multizábal, A. (1926). Monografía geográfica e histórica del Archipiélago de Chiloé.

Tornero, R. S. (1872). Chile ilustrado. Chile, Valparaíso: Librerias i Agencias del Mercurio.

Torrealba, A. (1917). Tierras fiscales e indigenas su legislación y jurisprudencia. Santiago: Editorial Imprenta
Universitaria.

Urbina B., R. (1983). La periferia meridional indiana: Chiloé en el siglo XVIII. Chile, Santiago: Ediciones Universitarias de Valparaíso.

Urbina B., R. (2004). Población indigena, encomienda y tributo en Chiloé, 1567-1813: política estatal y criterios locales sobre el servicio personal de "veliches" y payos. Valparaíso: Instituto de Historia, Pontificia Universidad Católica de Valparaíso.

Urbina, R. (2009). El modo de comerciar de los chilotes a fines del siglo XVIII. Revista Cultura de y desde Chiloé, 23, 65-95.

Urbina Carrasco, M. X. (2011). Análisis histórico-cultural del alerce en la Patagonia septentrional occidental, Chiloé, siglos XVI al XIX”, Magallania, 39-2, 57-73.

Urbina Carrasco, M. X. (2013). "La situación de Chiloé durante las guerras de independencia”. En S. O'Phelan \& G. Lomné (Eds.), Abascal y la contra-independencia de América del Sur, Institut Francais d'Etudes Andines \& Pontificia Universidad Católica del Perú (Lima), 187-226.

Urbina Carrasco, M. X. (2016a). Interacciones entre españoles de Chiloé y Chonos en los siglos XVII y XVIII: Pedro y Francisco Delco, Ignacio y Cristóbal Talcapillán y Martín Olleta. Chungará, 48-1, 103-114.

Urbina Carrasco, M. X. (2016b). De la conquista a la república”. Aldunate, Carlos (Eds.), Santiago, Chiloé: Museo Chileno de Arte Precolombino.

Willebald, H. (2015). Distributive patterns in setter economies: agricultural income inequality during the First Globalization, 1870-1913. Historia Agraria, 66, 75-104.

Williamson, J. G. (2010). Five centuries of Latin American income inequality. Journal of Iberian and Latin American Economic History, 28-02.

Yudilevich, D., \& Castro Le-Fort, E., (Eds.) (1995). Darwin en Chile (1832 - 1835). Viaje de un naturalista alrededor. Chile, Santiago: Editorial Universitaria. 
M. LLORCA-JAÑA et al.

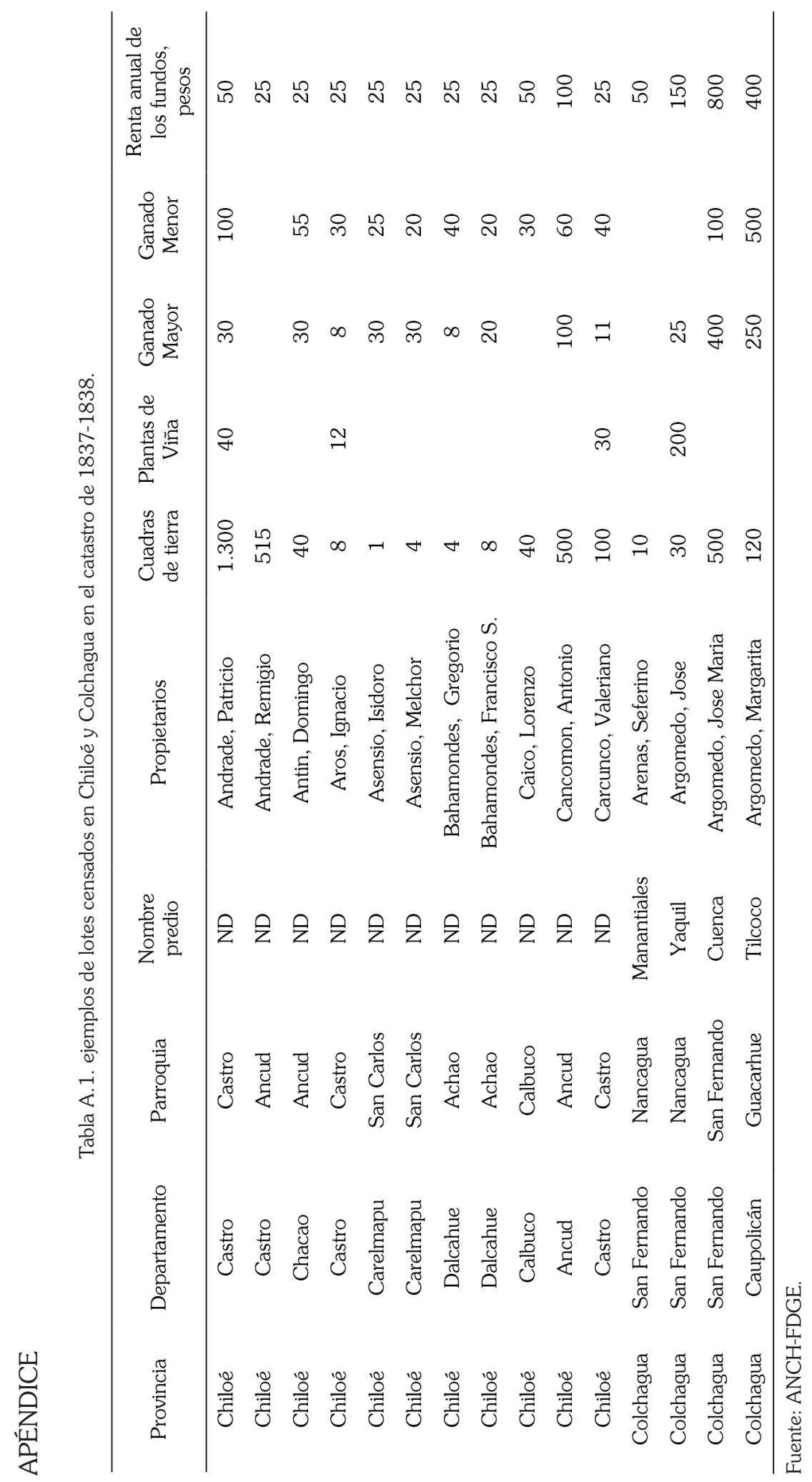


Tabla A.2. Ejemplos de lotes censados en Chiloé en el catastro de 1852.

\begin{tabular}{lcccc}
\hline Provincia & Departamento & $\begin{array}{c}\text { Renta anual de } \\
\text { los fundos, pesos }\end{array}$ & Nombre predio & Propietarios \\
\hline Chiloé & Castro & 90 & Quelquel & Aguilar, Valentín \\
Chiloé & Chacao & 28 & Caulin & Alarcón, Francisco \\
Chiloé & Castro & 30 & Tutuy & España, Manuel \\
Chiloé & Chacao & 36 & Lliuco & Guenchur, Antonio \\
Chiloé & Ancud & 36 & Puquetrico & Guenul, Severino \\
Chiloé & Castro & 40 & Nercon & Marimán, Mateo \\
Chiloé & Calbuco & 25 & Fuerte & Martínez, María Marta \\
Chiloé & Ancud & 70 & Piticura & Martínez, Santiago y padre \\
Chiloé & Carelmapu & 30 & Guatrunes & Martínez, Serafín \\
Chiloé & Chonchi & 36 & Sin Nombre & Melipillán, Juan Miguel \\
Chiloé & Lemui & 44 & Detif & Melipillán, Trinidad \\
Chiloé & Achao & 26 & Gueno i Achao & Mella, Javier \\
Chiloé & Achao & 39 & Sin Nombre & Mella, Lorenzo \\
Chiloé & Achao & 25 & Gueno i Achao & Mella, Luis \\
Chiloé & Dalcahue & 46 & Chaurahue & Perán, Justo \\
\hline
\end{tabular}

Fuente: Chile 1855. 
